\title{
Text dependent speaker identification system based on deep learning
}

\author{
Yusra Faisal Al-Irahyim ${ }^{*}$, Qasim Sadiq Mahmood ${ }^{2^{*}}$ \\ ${ }^{1 *, 2}$ Department of Computer Science, Collage of Computer Science and Mathematics, University of Mosul, \\ Mosul, Iraq \\ E-mail: 1*yusrafaisalcs@ uomosul.edu.iq, ${ }^{2}$ Qasim.csp56@student.uomosul.edu.iq
}

(Received May 16, 2021; Accepted July 03, 2021; Available online September 01, 2021)

DOI: 10.33899/edusj.2021.130144.1161, (C 2021, College of Education for Pure Science, University of Mosul.

This is an open access article under the CC BY 4.0 license (http://creativecommons.org/licenses/by/4.0/).

\begin{abstract}
Speaker identification techniques are one of those most advanced modern technologies and there are many different systems had been developed, from methods that used to extract characteristics and classification. The applications of Speech identification are quite difficult and requires modern technologies with a large number of audio samples and resources.

In this research, the system of speaker identification had been designed based on a text (the word or sentences are pre-defined) which give the system the capability to identify the speaker in the least time, number of training samples and resources. The system consists four main parts, the first one is to create audio databases. In the study, two audio databases were relied upon, the first being a database (QSDataset) and the second database (audioMNIST_meta). The databases were processed and configured in a way that was explained in the body of the research later. The second part of the research is to extract the characteristics through the pitch coefficients algorithm, while the third part is the use of the neural network as a classifier. And the last part of the research is to verify the work and results of the system. The test results showed the ability of the MNN network to deal with the smallest number of data, as it achieved a percentage of $100 \%$. As for large data, it ranged from $80 \%$ to $81 \%$. Unlike CNN network, the results were not good for the few data, from $60 \%$ to $76 \%$, and with large data it was The results are excellent, from $91 \%$ to $96 \%$.
\end{abstract}

Keywords: Speaker identification, MFCC, Multilayer Neural Network (MNN), convolution neural network (CNN), Deep Learning

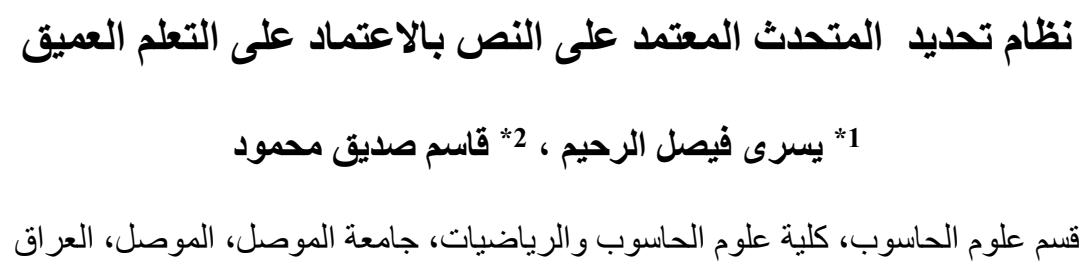




\section{Journal of Education and Science (ISSN 1812-125X), Vol: 30, No: 4, 2021 (141-160)}

تعد تقنيات التعرف على المتحدث من أهم التقنيات الحديثة وقد طورت العديد من الأنظمة المختلفة من حيث الطرق المستخدمة في استخراج الخصائص وطرق التصنيف. تشكل تطبيقات التعرف على المتحدث تحديًا كبيرًا، حيث يتطلب تقنيات حديثة وعدد كبير من عينات الصوتية وموارد كبيرة. في هذه البحث صمم نظام التعرف على المتحدث المعتمد على النص (تكون الكلمة او الجمل محددة مسبقا)حيث يتميز النظام بقدرته على التعرف على المتحدث باقل وقت واقل عدد من عينات التدريب واقل الموارد. يتكون النظام من اربعة اجزاء رئيسة وهي: الجزء الأول بناء قواعد بيانات صوتية, واعتمد في الدراسة على قاعدتين للبيانات الصوتية, الاولى قاعدة بيانات (QS-Dataset) وقاعدة البيانات الثانية(audioMNIST_meta)، ،وقد تم معالجة و تهيئة قواعد البيانات بطريقة تم شرحها في متن البحث لاحقا. والجزء الثاني من البحث استخلاص الخصائص عن طريق خوارزمية معاملات درجة النغم اما الجزه الثالث فهو استخدام الشبكة العصبية كمصنف. واما الجزء الاخير في البحث فهو التحقق من عمل و نتائج النظام. اوضحت نتائج الاختبار قدرة شبكة MNN على التعامل مع اقل عدد من البيانات حيث حقت نسبة (100\%) واما مع البيانات الكبيرة تتراوح من 80\% الى 81\% على عكس شبكةCNN كانت نتائج غير جيدة بالنسبة للبيانات القليلة من60\% الى 76 \% ومع بيانات

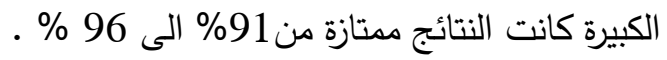

الكلمات المفتاحية: التعرف على هوية المتحدث ،MFCC ، الشبكة العصبية متعددة الطبقات (MNN)، الشبكة العصبية الالتفافية •

1 1

الصوت عبارة عن إشارة مصنوعة من نغمة (tone) أو عدد من النغمات المتصلة ببعضها البعض لتعني شيئًا ما وتستخدم للتواصل بين البشر أو أي كائن حي ، حيث يعبرون من خلالها عما يريدون قولاً أو فعلاً بوعي أو بغير وعي ، والإحساس الناجم عن ذلك موجات تسمى السمع. بسبب الصوت ، يحصل الناس على العديد من الخبرات في الحياة. في الماضي ، لم يكن الصوت الذي يصدره الإنسان هو الطريقة الوحيدة المستخدمة للتواصل مع بعضهم البعض ، ولكنهم استخدموا أيضًا العديد من الأشياء التي تصدر ضوضاء واهتزازات

مثل الطبول والمزامير [1].

وفقًا لـ Gray و M. S. Saito تم اقتراح الأفكار الأولى لاستخدام أنظمة التعرف على المتحدث في عام 1966 بواسطة Itakura

استحوذت شركة Nuance Communications على شركة . تلطوير طريقة جديدة تعتمد على الخصائص البيومترية[2]. يمكن تقسيم تقنية التعرف على المتحدث تقريبًا إلى مجالين فرعيين ، وهما التعرف على الكلام والتعرف على المتحدث [3]. التعرف على الكلام هو نهج لتحليل محتويات الكلمات / الكلام الذي يتحدث به المتحدث. يستخدم كل نظام من أنظمة التعرف على الكلام العديد من الخوارزميات لتحويل الموجات الصوتية إلى بيانات مفيدة للمعالجة والتي يتم تفسيرها بعد ذلك بواسطة الجهاز ـ هذه الأنظمة تتتج بعد ذلك

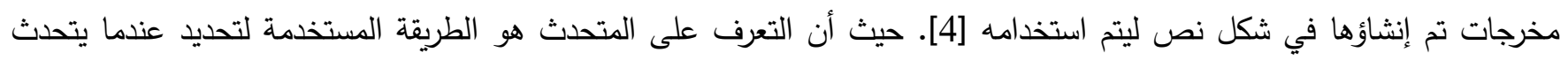
المتحدث بهذه الكلمات / الكلام. تحاول تقنيات التعرف على المتحدث تغطية الجوانب المختلفة للتعرف على الأشخاص من خلال أصواتهم. لأن كل متحدث له طريقته المميزة في التحدث ، بما في ذلك استخدام لهجة معينة ، والإيقاع ، وأسلوب التتغيم ، ونمط النطق تلقي 
، واختيار المفردات ، وما إلى ذلك، يمكن تقسيم أنظمة التعرف على المتحدث إلى نوعين المعتمدة على النص والمستقلة عن النص. في

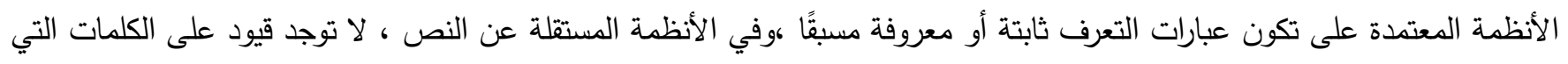

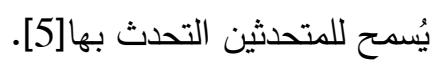
تتمثل الصعوبة الرئيسية لنظام التعرف على المتحدث في أنه من المستحيل نطق كلمة بالطريقة نفسها تمامًا في وقتين مختلفين. يعتمد ذلك على مدى سرعة نطق الكلمة ، ويمكن أن تختلف النغمة [2]. إنّ نظام تمييز المتحدث هو نظام يعتمد على الكلام إذ يتم تحليل إثارة الكلام للحصول على فلى ميزات أقل تتوعًا وأكثر تمييزًا ، ولاستخراج هذه القيم يتم استخدام الطرق مختلفة تسمى ميزات إثارة الكلام (Feature extraction)، ويتم استخدام الميزات التي تم الحصول علئ علئها

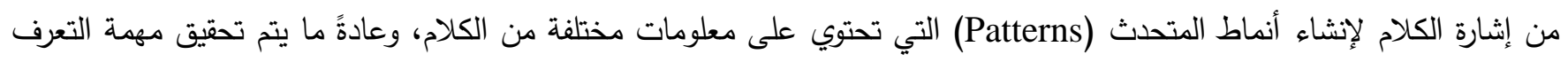
على المتحدث من خلال الحصول على إشارة الكلام واستخراج الميزة و نمذجة ميزات الكلام ومطابقة الأنماط والحصول على دألى درجة المطابقة المطلوبة. وتُعد الطرق التعلم العميق (Deep learning) أحد أهم التقنيات المستخدمة لتصميم مختلف أنظمة تمييز الأنماط في مجال معالجة إشارة الصوت. الهدف من هذه الدراسة هو بناء نموذج نظام يمكن استخدامه في عديد من تطبيقات (البنوك وبالانظمة الأمنية ...الخ) حيث يقوم النظام بالتعرف على المتحدثين من خلال الخصائص الفيزيائية الفريدة لكل صوت وتتتقل من الطرق التقليدية إلى الطرق أكثر صرامة وموثوقية في تحديد هوية المتحدث. لهذا السبب، تلعب المعالجة الرقمية لإشارة الكلام والطريقة التي سيتعامل بها النظام مع الأصوات الواردة

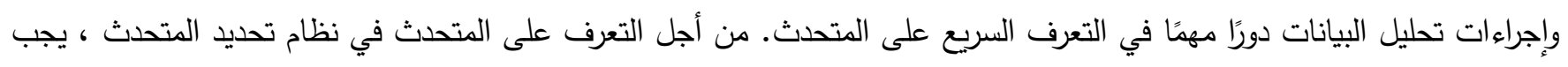
أن تمر الإشارة عبر عدة مراحل منها كما في الثكل (1): 2. 1 3. استخراج الخصائص و معالجة الاشارة

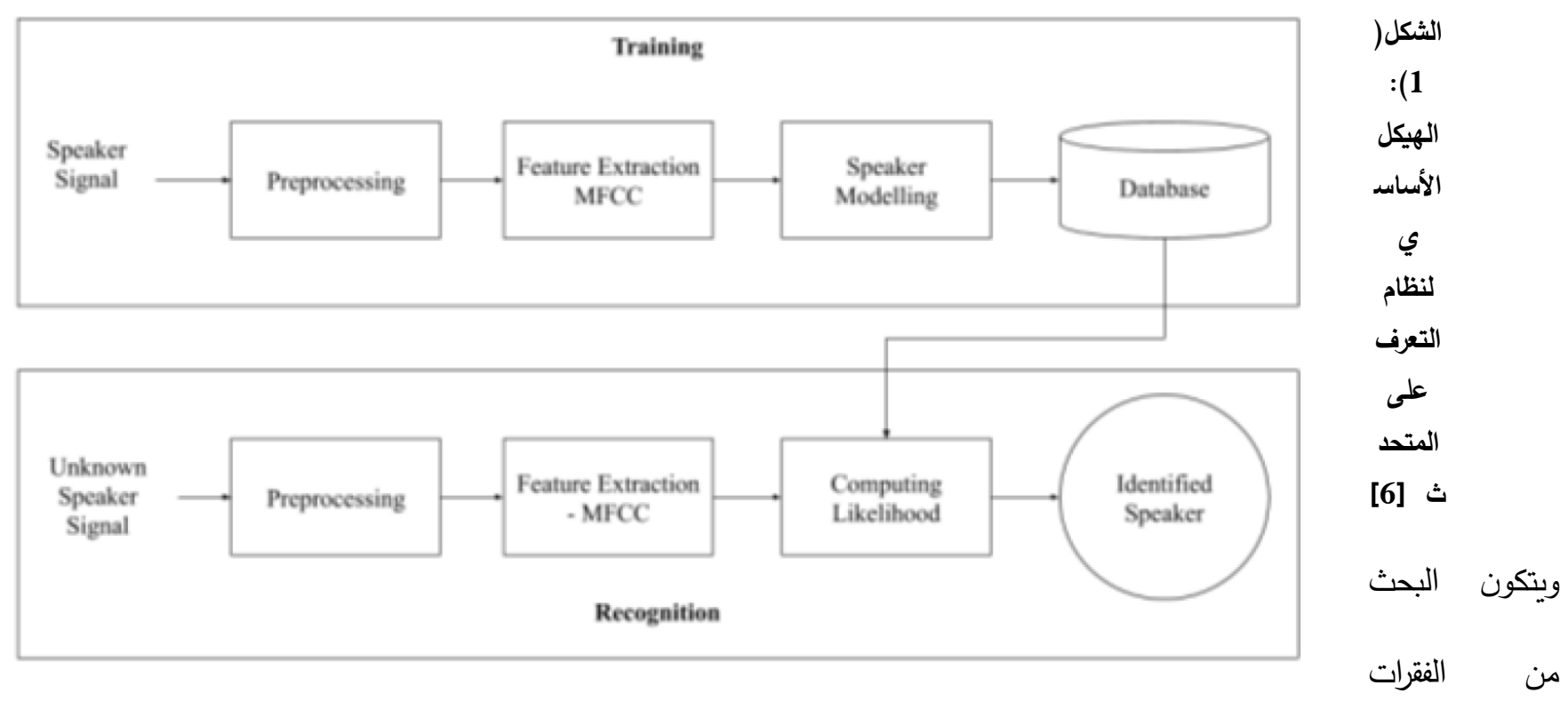

الرئيسية التالية: 


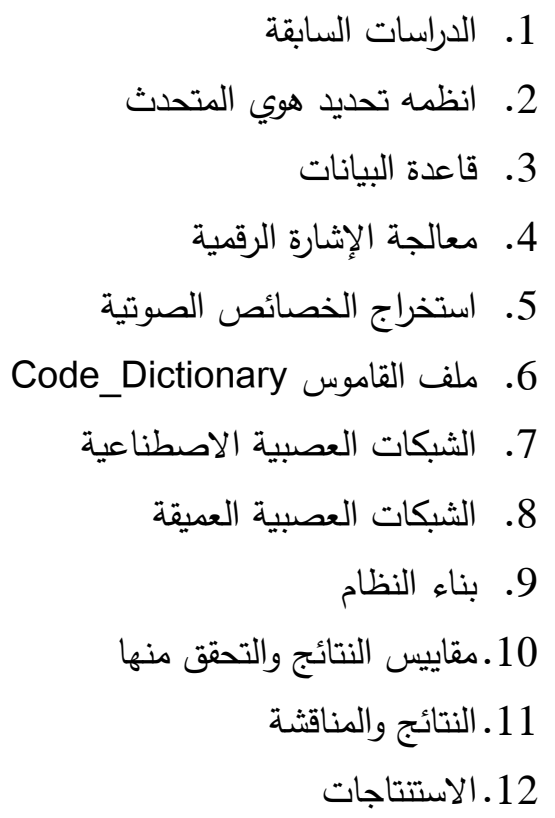

2. الدراسات السابقة

جذبت تقنيات التعرف على المتحدث العديد من الباحثين على مدار الاعوام الماضية وذللك لما لها من تطبيقات مهمة في مختلف تصف المجالات، أن التعرف على المتحدث تعد أحد اهم الخطوات في العديد من التطبيقات منها تحسين التعرف على المتحدث في نظام البنوك وتطبيقات التعرف الالي للتكلم وغيرها، ولا سيما في وجود التطورات التكنولوجية الحديثة ومعالجة الاشارة والتعلم الالي، وفيما

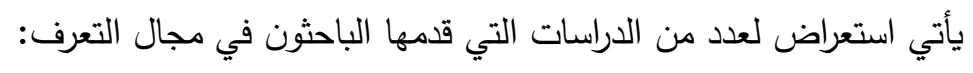
• في سنة 2015 قام كل من ول Diana Mulitaru و Inge Gavant بدراسة المصنفات المختلفة في التعرف سواء التعرف على الكلام او التعرف على المتحدث.في هذه الدراسة تم الاعتماد على قاعدة بيانات المتاحه تجاريا (TIMIT) لفحص المصنف،كما تم استخلاص الخصائص عن طريق دمج خوارزمتي التتبؤ الخطي الإدراكي perceptual linear sprediction(PLP)) ماركوف المخفي بنسبة 10\% [6]. في عام 2016 قام كل من Poonam Sharma و Angali Garg و بدارسة تبين معدل التعرف على كلمات اللغة الهندية وتأثير الجنس ونوع الشبكة العصبية وخوارزمية استخلاص الخصائص على نسبة التعرف. تبين في الدارسة أن نسبة التعرف

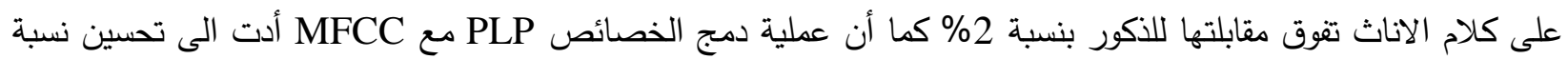

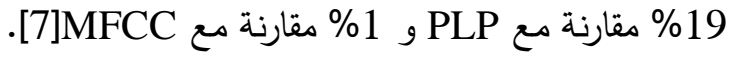

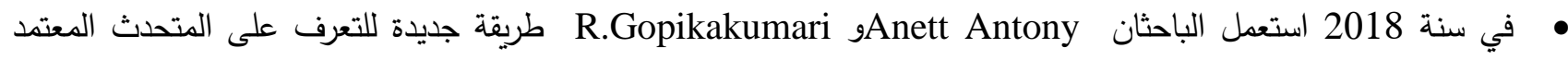
على النص و الغير معتمد على النص بستخدام الشبكة بيرسبترون متعددة الطبقات (MLP)( كاداة تصنيف ،حيث تتميز باقل تعقيد. تم تقليل تعقيد الشبكة من خلال استخراج الخصائص للإشارة الصوتية عن طريث دمج خوارزمية درجة النغم (MFCC ) و خوارزمية التحويل الحقيقي الفريد من نوعها ( Unique mapped real transform ) توصلت الدراسة انه عند دمج الخوارزميات تكون نتائج استخراج الخصائص افضل بكثير مما إذا استخرجت (UMRT) 
الخصائص عن طريق فقط خوارزمية درجة النغم (MFCC).واستخدمة في هذه الدراسة قاعدة بيانات مكونة من 7 انات و 8

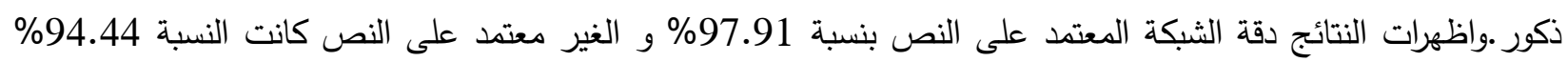

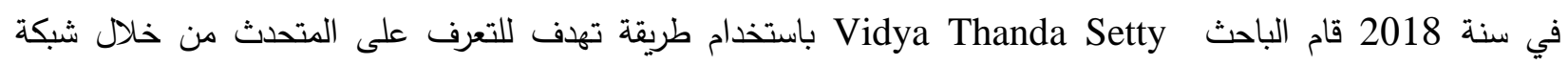
العصبية العميقة (DNN)(تقوم هذه الدراسة الى تقليل التعقيد الموجود في شبكة العصبية

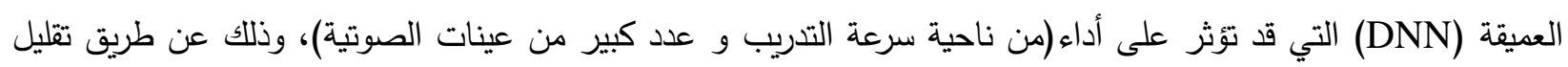

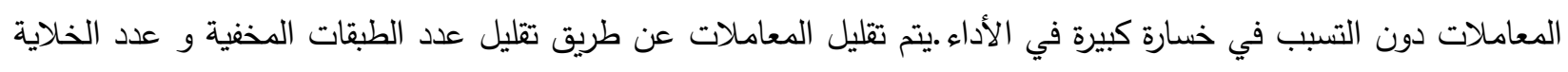

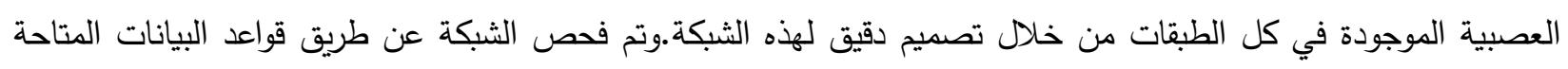

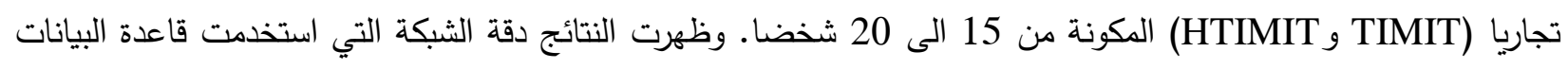

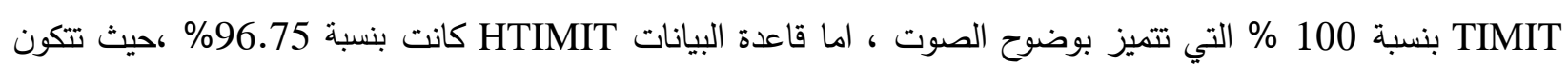

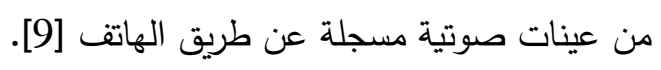

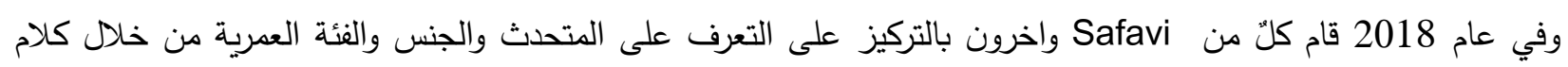

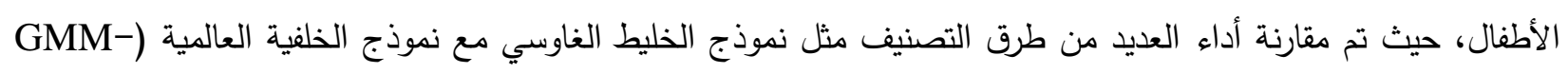
(Universal Background Model (GMM-UBM)

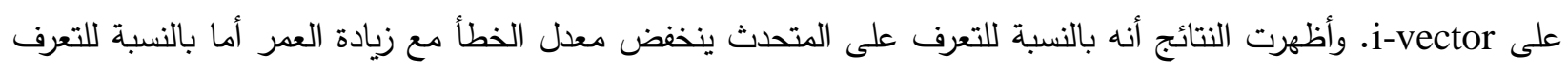

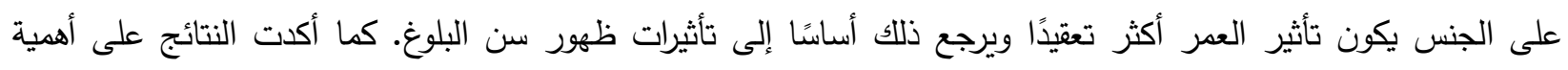

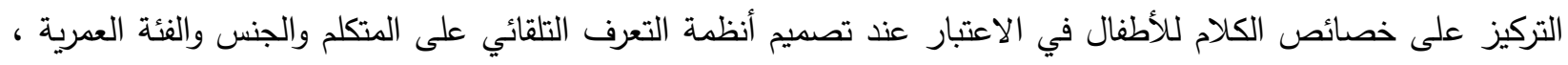

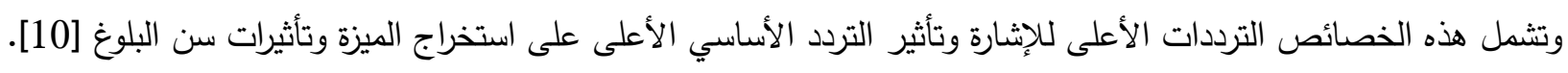

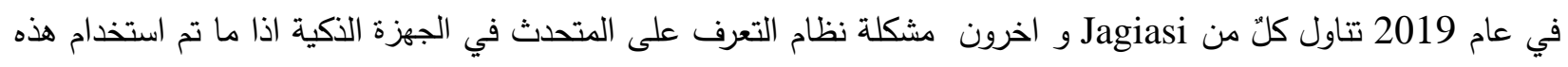

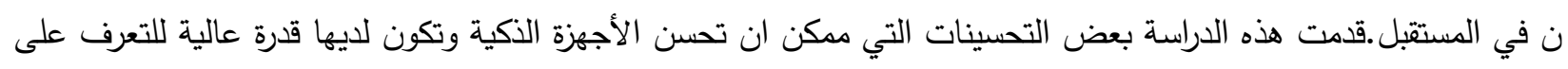

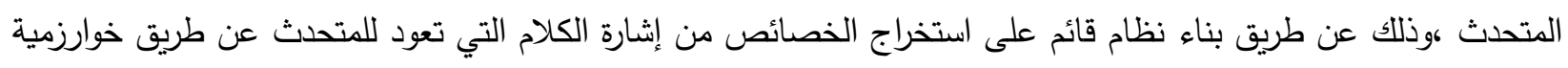

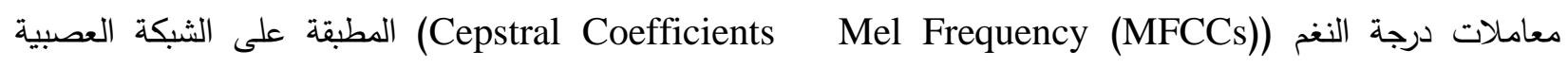

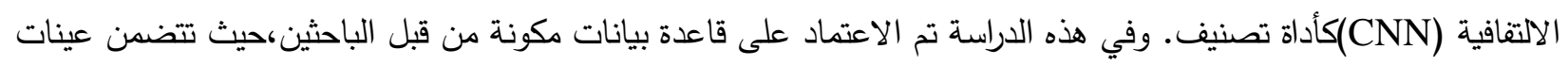

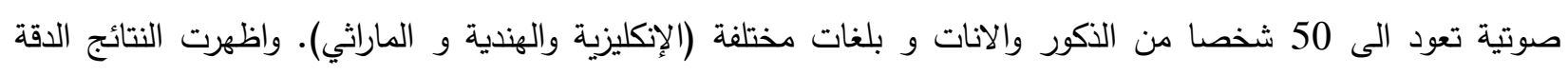

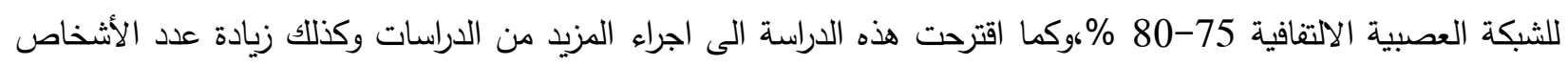
في عملية تدريب الثبكة [11]. في عام 2020 قامت وآخرون بدراسة التعرف على المتحدث في ظل ظروف مختلفة من حيث

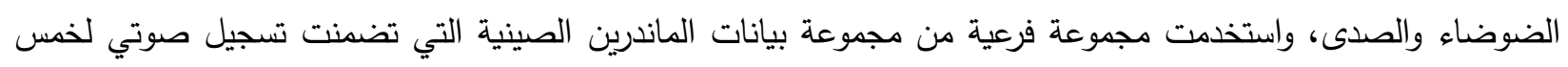

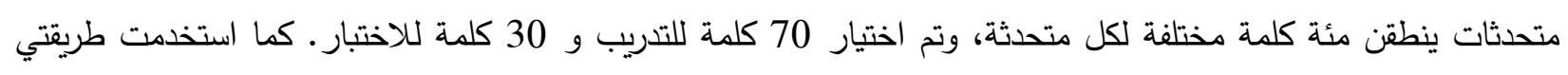

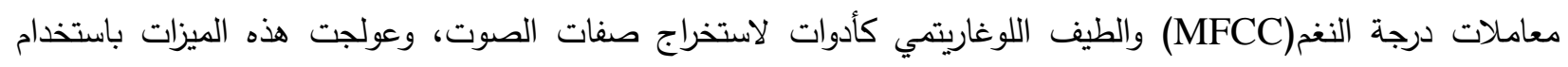

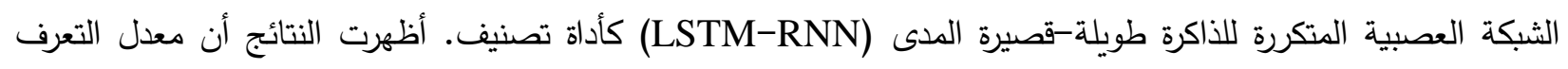

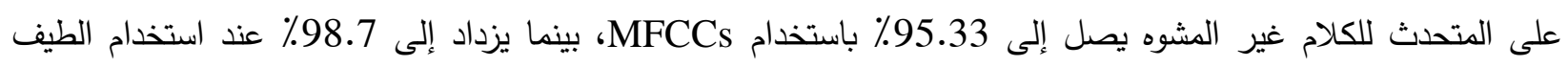


اللوغاريتمي، بينما أظهرت النتائج بالنسبة للكلام المشوه أن معدل التعرف على المتحدث يصل إلى 90\% باستخدام طريقة الطيف [12].

اثبتت الدراسات السابقة ان بناء أي نظام للتعرف على المتحدث يعتمد بشكل أساسي على احدى خوارزميات ) MFCC (DLP,UMRT او الدمج بين هذه الخوارزميات لاستخلاص الخصائص واحدى الشبكة العصبية (DNN,CNN,RNN) كاداة تصنيف.كما توصل الباحث انه لا يمكن الجزم بوجود خوارزمية افضل من غيرها لان دقة التعرف ترتبط بطبيعة اللغة والجنس المتحدث

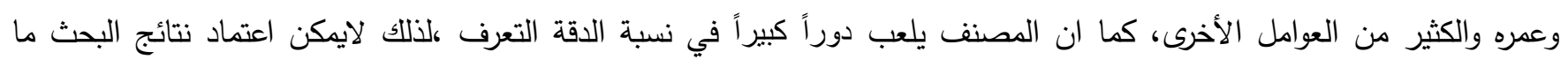
كنتائج شاملة وذلك بسبب التتوع الكبير جدا في ظروف عملية التعرف ومحدداتها.

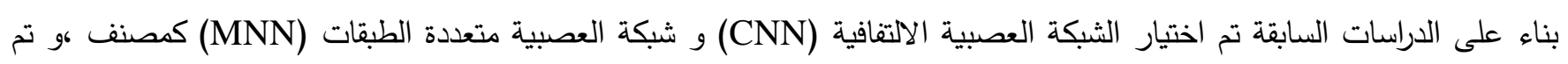
اختيار خوارزمية درجة النغم (MFCC) في استخلاص الخصائص.

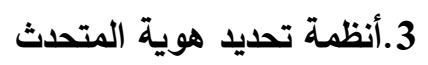
تتطلب أنظمة التعرف على هوية المتحدث عادةً العديد من العمليات والمراحل التي يجب أن تمر فيها الإشارة الصوتية للوصول إلى النتيجة. هناك فئتان من هذه الأنظمة التي تستخدم للتعرف على هوية المتحدث منها الانظمة المستقلة عن النص أو الأنظمة التي تعتمد على النص. في الأنظمة التي تعتمد على النص، حيث تكون الكلمة او الجمل معروفة مسبقا. في الأنظمة المستقلة عن النص، لا توجد قيود على الكى التهل

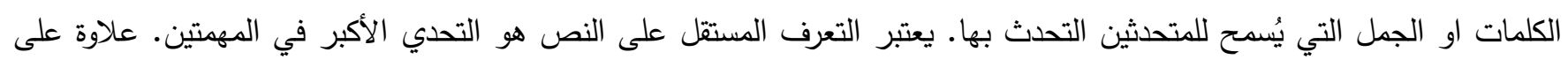
ذللك، في الحياة الواقعية، تعتبر الأنمة المستقلة عن النص أكثر اهتماما من الناحية التجارية من الأنظمة المعتمدة على النص لأنه من الصعب تقليد عبارة غير معروفة أكثر من تلك المعروفة.في هذه الدراسة تم بناء النظام من نوع معتمد على النص. 4. قواعد البيانات

تشكل قواعد بيانات الصوتية الركن الرئيسي في بناء نظم حاسوبية، وتشكل البنية التحتية لبناء نظم تخاطب مع الحاسوب. في هذه الدراسة تم الاعتماد على اثثين من قواعد بيانات الاولى (audioMNIST_meta) تم الحصول عليهل من موقع (Kaggle) ،حيث تم تكوين قاعدة البيانات من قبل مجموعة من الباحثين[13] . اما قاعدة البيانات الثانية تم تكوينها من قبل الباحث (QS-Dataset). تتكون قاعدة البيانات (QS-Dataset) من متحدثين باللغة العربية اما قاعدة البيانات (audioMNIST_meta) فتضم متحدثين باللغة الإنكليزية، وذلك من اجل شمولية عمل النظام على جميع الغات.

تتألف قاعدة البيانات (QS-Dataset) من ملفات صوتية احادية القناة لـ 35 شخص، تضم 25 مقطع صوتي لكل متحدث بالامتداد 22050. خلال عملية التسجيل يعمل كل متكلم على تكرار لجملة معينة بمقدار 25 مرة في تسجيل صوتي واحد، ومن ثم خزن التسجيل الصوتي التابع لمتكلم معين في ملف مستقل عن بقية المتكلمين. الجملة التي تم اختيارها هي (سبحان الله وبحمده سبحان الله العظيم) وذللك لضمان الحصول على فترة زمنية أطول. قاعدة البيانات (audioMNIST_meta) تتألف من 35 شخص، لكل شخص 25 مقطع صوتي. الكلمة التي تم اختيارها هي (One) في كل مقطع يتم نطقها ثلاثة او أربع مرات لضمان حصول على مدة زمينه كافية للتساوي مع قاعدة البيانات الأولى. 
تعرف الاشارة على انها كمية فيزيائية (مادية) متغيرة بتغير الزمان والمكان بالإضافة الى بعض المتغيرات المستقلة الاخرى، اما معالجة الاشارة فهي عملية استخراج معلومات مهمة من الاشارة بطريقة فعالة وقوية. ومن الاشارات المهمة في التواصل بين البشر هي اشارة الكلام، فعندما يتكلم الانسان فإنه ينشئ موجة تتاظرية في الهواء تحول عن طريق اللاقط الصوتي الى اشارة تتاظرية ( Analog) signal بينما تحول الى بيانات رقمية عندما تخزن على جهاز الحاسوب بشكل (0,1)[14]. ان أحدى الخطوات الرئيسية في معالجة وتحليل الاشارات الصوتية تتمثل بتحويل الاشارة الخام الى تمثيلات تتاسب الهدف المطلوبة انجازه، يتم تمييز موجة صوتية عن اخرى من خلال بعض الخصائص منها التردد (Frequency) والسعة (Amplitude) والطور (Phase)[13]. 1.5

يمكن القول بأن مرحلة استخلاص الخصائص هي من أهم مراحل التعرف على الكلام؛ لما لها من تأثير على نسبة التعرف الكلية للشبكة العصبية الاصطناعية. نورد فيما يلي واحدة من أهم الخوارزميات المستخدمة في استخلاص الخصائص والتي تسمى خوارومية درجة

\section{Mel Frequency Cepstral Coefficients (MFCC) 1.1 .5}

تعتبر خوارزمية MFCC من أهم خوارزميات استخلاص الخصائص وذلك بسبب النتائج الجيدة التي تعطيها. يوضح الشكل(2) مخطط يمثل الخطوات الاساسية لخوارزمية MFCC ، اذ تبدأ بالمعالجة الأولية لإشارة الكلام، ثم تقطيع الإشارة الى اطارات قصيرة الزمن، يلي ذلك عملية الضرب بالنافذة ثم تطبيق تحويل فورير على كل إطار ، بعدها يتم تحويل الترددات الى ترددات Mel ومن ثم تجمع الطاقة الإساته

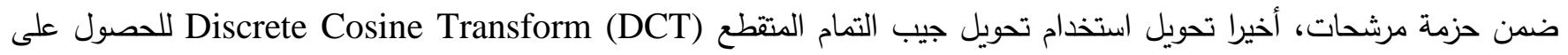
المعاملات.

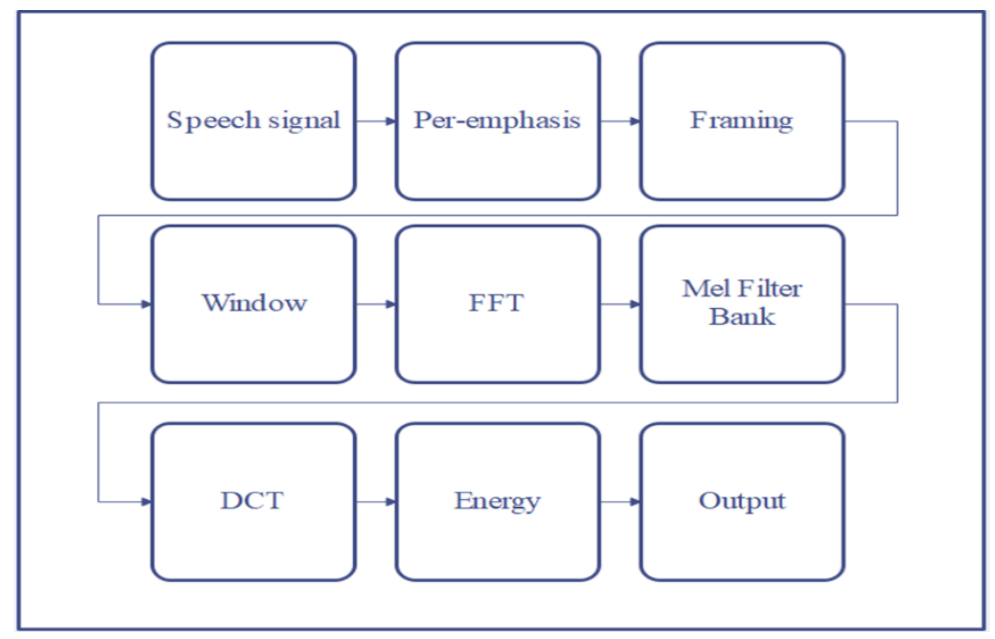

[1]MFCC الشكل (2): مخطط لمراحل

Pre-emphasis 1.1.1.5 التشديد المسبق

high بعملية MFCC وهي عملية مرشح تردد عالي Pre-emphasis وهثل الخطوة الاولى من استخلاص خصائص الاشارة بواسطة pass filter عالي التردد) كما موضح في الثكل (3)، حيث يتم إعادة تقييم كل قيمة في إشارة الكلام باستخدام المعادلة(1) التالية: 
$\mathbf{S 2}(\mathbf{n})=\mathbf{s}(\mathbf{n})-\mathbf{a}^{*} \mathbf{s}(\mathbf{n}-1) \ldots$

حيث تمثل (n) إثارة الكلام،s2(n) الاشارة الناتجة من عملية ال pre-emphasis، اما a فهي قيمة ثابتة تتراوح بين (0.9 و 0.1).
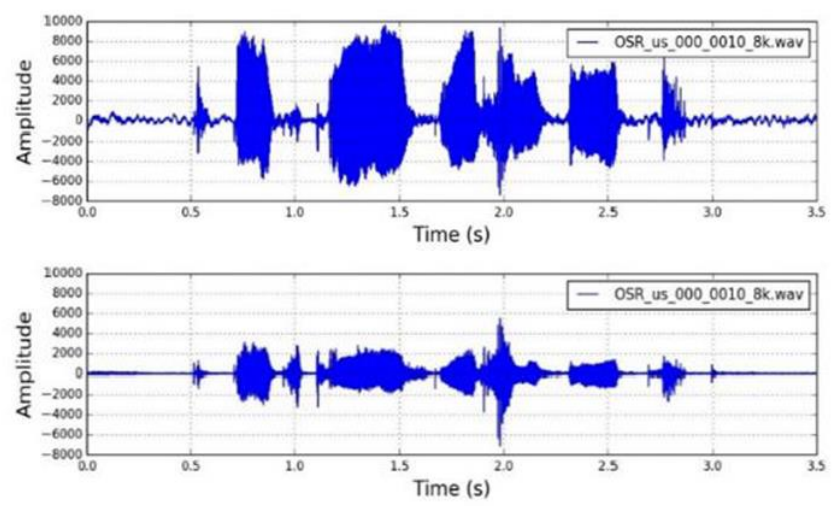

[1]Pre-emphasis الثكل (3): الإشارة قبل وبعد عملية

2.1.1.5

نظرا لكون الإشارة الصوتية متغيرة باستمرار في الكلام. يتم نمذجة هذه الإشارة المتغيرة عن طريق إنثاء مقاطع صغيرة مأخوذة من الصوت على أنها ثابتة، وذلك من خلال عملية التأطير وهي عملية فصل العينات من الصوت الخام إلى مقاطع ذات طول ثابت

يشار إليها بالإطارات (Frames) وعادة ما يكون طول الاطار 20ms [1]. بعد تهيئة الإطارات يتم تمرير كل اطار من خلال نافذة بفترة معينة وذلك لتقليل عدم استمرارية الإشارة في بداية ونهاية كل اطار ( تقليل انقطاع إشارة الكلام قبل وبعد كل إطار) كما موضح في الشكل (4) [15]. اذا عرفنا النافذة بـ W(n) والاشارة الداخلة بـ XX (n) فان الإشارة الخارجة هي : (1)

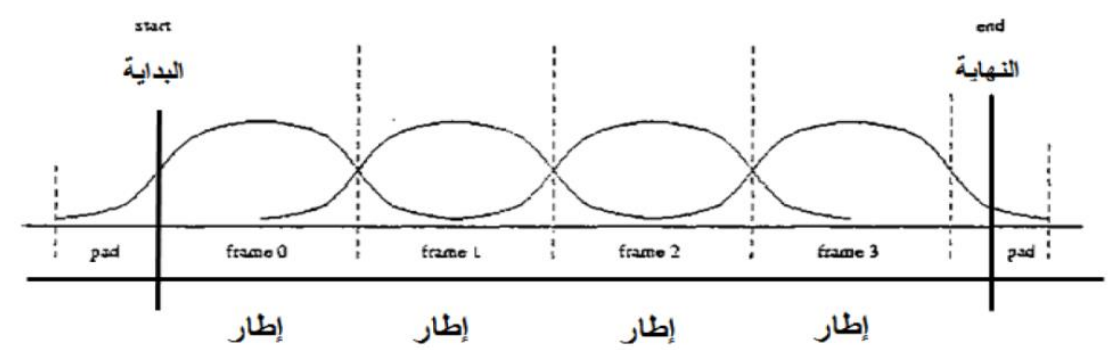

الثكل (4) نموذج لعملية الإطارات والنوافف

$X_{1}^{\prime}(n)=X_{1}(n) * W(n) \quad 0 \leq n \leq N-1$ 
هنآك العديد من أنواع النوافذ مثل: نافذة هامنغ (Hamming window) ونافذة هاننغ (Hanning window) ونافذة ) Blackman Kaiser (window والنافذة المستطيلة (Rectangular window) والنافذة المثلثة (Triangular window) ونافذة القيصر (R) (window) ونافذة غاوس (Gaussian Window) ونافذة ويلش (Welch Window). النافذة الأكثر شيوعا في أنظمة التعرف على هوية المتحدث هيا نافذة هامينغ (Hamming window) والمعروفة بالمعادلة (3): $w(n)=0.54-0.46 \cos \left(\frac{2 \pi n}{N-1}\right) \quad 0 \leq n \leq N-1 \ldots$

حيث تمثل w(n) العينة الجديدة، n هي تسلسل العينة المفردة وN هو الطول الكلي النافذ.

Fast Fourier Transform (FFT) 3.1.1.5 تحويل فورير السريع بعد عملية الضرب بالنافذة، يتم تطبيق خوارزمية تحويل فورير السريع لكل إطار، وذللك لاستخراج مركبات التردد للإشارة في مجال الزمن [1].كما موضح في الشكل (5).
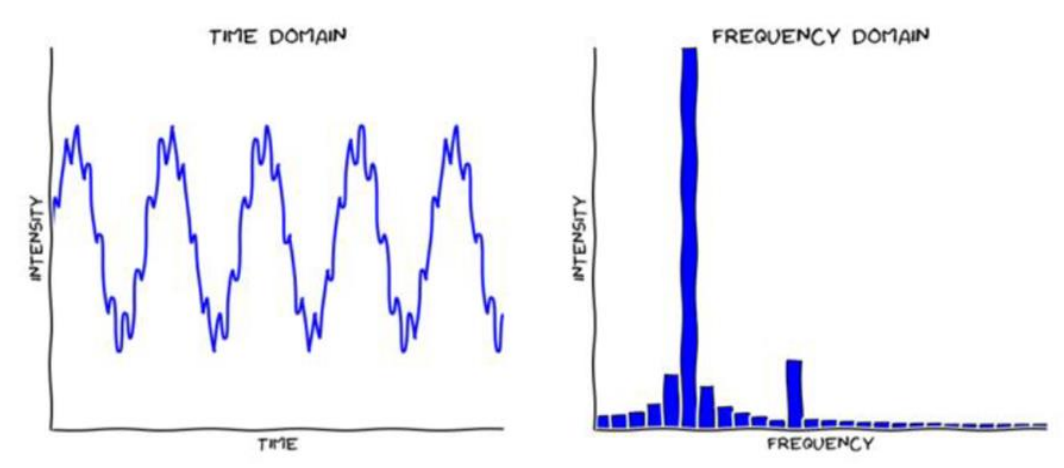

[1](FFT) الثكل (5) يوصف التحويل فورير السريع

Mel Filter Bank بنك المرشحات الميل 4.1.1.5

وهي عبارة عن مجموعة من مرشحات التي تحاكي النظام السمعي البشري. بدلاً من اتباع مقياس خطي، تعمل هذه المرشحات المثلثية اللوغاريتمية عند الترددات الأعلى والخطية عند الترددات المنخفضة، وهو أمر نموذجي في إثارات الكلام. عادة ما يحتوي بنك المرشح على 40 مرشحًا وكما موضح بالثكل (6). يمكن أن يتم التحويل بين نطاقات Mel (m) و (f) (fertz من خلال: $m=2595 \log _{10}\left(1+\frac{f}{700}\right)$

$f=700\left(100 \frac{m}{2595}-1\right)$

يمثل ناتج عملية الترشيح المجموع المرجح للترددات الطيفية التي تتوافق مع كل مرشح. تحدد هذه القيم ترددات الإدخال في مقياس ميل[16]. 


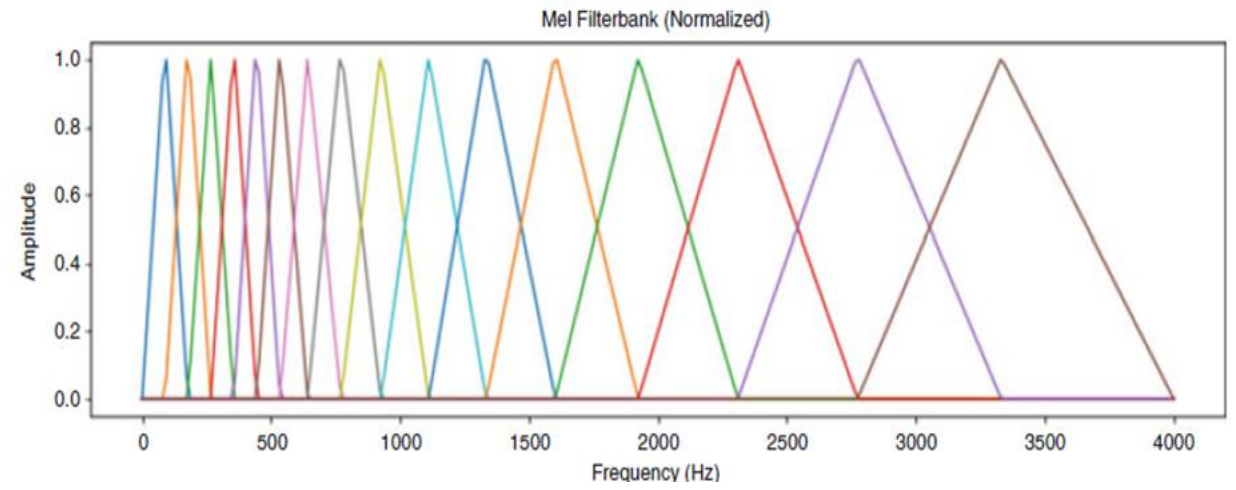

الثكل (6) بنك مرشح ميل[17]

Discrete Cosine Transform (DCT) 5.1.1.5 تحويل جيب التمام المنفصل

يقوم تحويل جيب التمام المنفصل (DCT) بتعيين ميزات مقياس Mel إلى مجال الوقت. الدالة DCT مشابهة لتحويل فورير ولكن يستخدم أرقام حقيقية فقط (تحويل فورير تتتج أرقام معقدة). وهو يضغط بيانات الإدخال في مجموعة من معاملات جيب التمام التي تصف التذبذبات في الدالة. ويشار إلى الناتج من هذا التحويل باسم MFCC كما موضح في الثكل (7). بعد استخلاص الخصائص يتم خزن النتائج بشكل (Vector) في ملف اسمه Code_Dictionary.

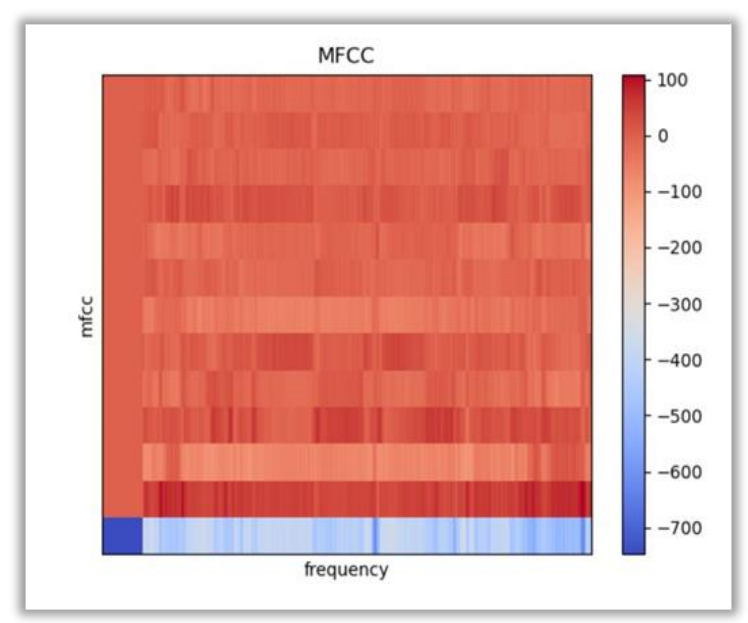

الثكل (7): التمثيل الطيفي لمعاملات درجة النغم

6. ملف القاموس Code_Dictionary عبارة عن ملف يتم فيه خزن النتائج بعد عملية استخلاص الخصائص حيث يتكون هذا الملف من ثلاثة حقول, الحقل الاول يضم أسماء المتحدثين والحقل الثاني يضم ارقام تمثل (labels) والذي يمثل فهرسة لهذه الاسماء المتحدثين والحقل الثالث يضم الخصائص لكل متحدث.يوضح الشكل(8) هيكلة الملف القاموس. 


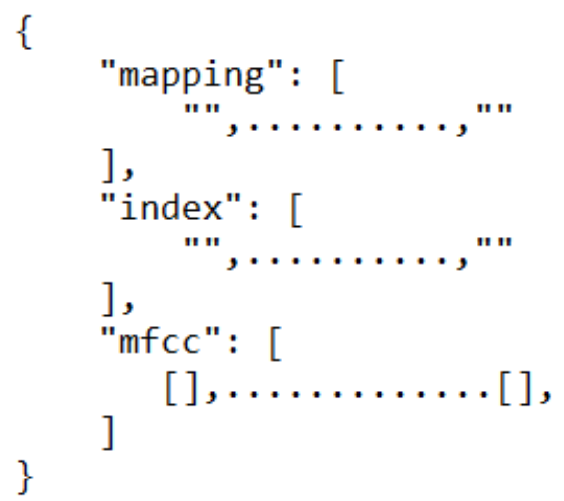

الثكل(8): هيكلة الملف القاموس

7. الثبكات العصبية الاصطناعية

تعرف الشبكات العصبية الاصطناعية (Artificial Neural Networks (ANN))على انها نماذج حسابية ذات قدرة على تجميع وتنظيم البيانات وتعلم المعلومات المعممة من خلال الاعتماد على المعالجة المتوازية. تتكون الثبكة العصبية من مجموعة من الطبقات (Layers) مكونة من وحدات معالجة تسمى بالخلايا (Neurons) متماثلة تتصل فيما بينها عن طريق ارسال الاشارات الموزونة الىى بعضها البعض، يتم في مرحلة التعلم تحديث المعاملات لتحقيق شرط معين وذلك عن طريق استخدام مجموعة التدريب (Learning Set) 1.7 الشبكة العصبية متعددة الطبقات تتألف هذه شبكة العصبية من طبقة واحدة او عدة طبقات من الطبقات المخفية. ان فائدة الطبقات المخفية هو اكتشاف المزايا (features) الامامي(forward-propagation) والانتشار العكسي(back-propagation). يتم الادخال المدخلات الى طبقة الادخال تمر الى طبقات المخفية ويتم المعالجة هذه الادخالات تما تمرر وتعالج مرة أخرى طبقة الإخراج تسمى هذه خوارزمية التدريب الانتشار الامام

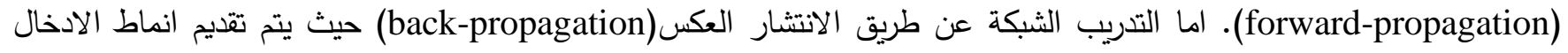
للتدريب إلى طبقة الادخال الشبكة. تقوم الشبكة بعد ذلك بنشر نمط الإدخال من طبقة إلى طبقة حتى يتم إنشاء نمط الإخراج بواسطة

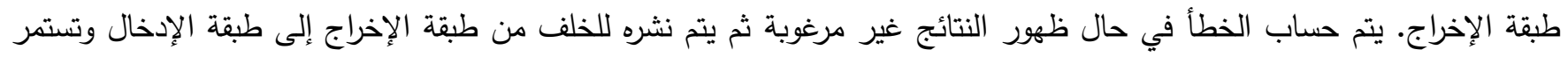
العملية لحين الحصول على النتائج المطلوبة [17]. 8.الثبكات العصبية العميقة أصبح التعلم العميق طريقة مثيرة للاهتمام وقوية لتعلم الآلة مع تطبيقات ناجحة في العديد من المجالات ، مثل معالجة اللغة الطبيعية ، والتعرف على الصور ، والتعرف على الأحرف المكتوبة بخط اليد، والتعرف على المتحدث ، ورؤية الحاسوب، حيث أظهر التعلم العميق مايق نجاحًا في التعرف على الكلام وتحديد هوية المتحدث على الطرق التقليدية مثل تلك التي تستخدم معاملات تردد ميل للتعرف على المتحدث باستخدام نماذج خليط غاوسي 
ان التعلم العميق يوفر طريقة اكثر تكيفا من خلال استخدام الثبكات العصبية العميقة التي تتعلم الخصائص من بيانات

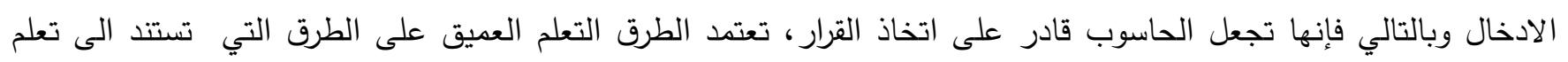
تمثيل البيانات .

1-8 - 1 الثبكة العصبية الاتنفافية

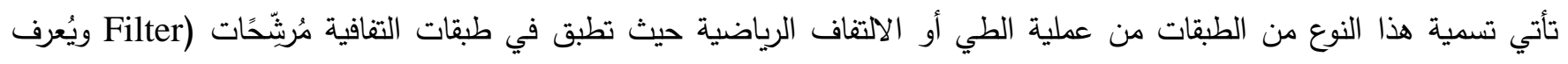

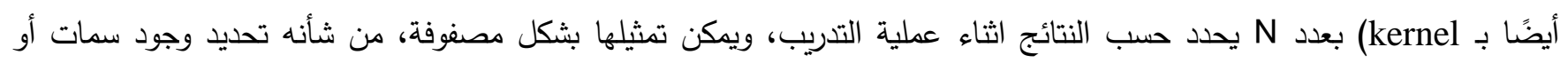

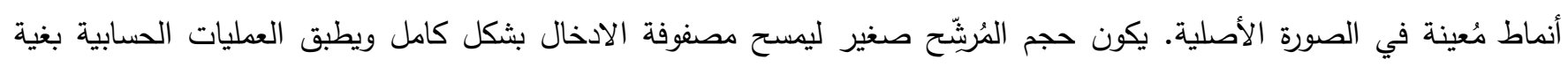

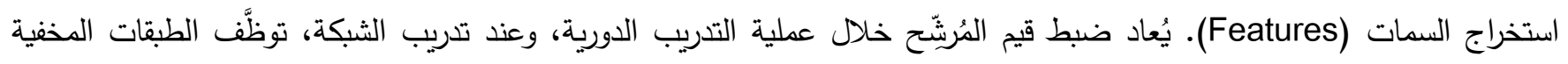

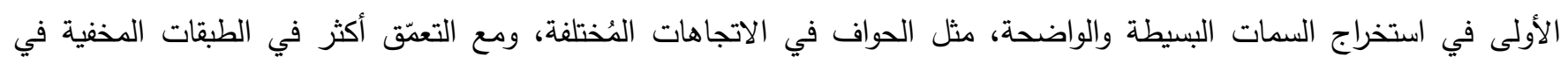

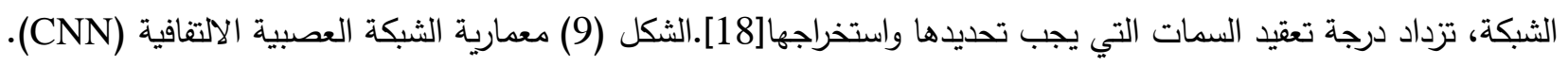
الشكل (9): نموذج الشبكة العصبية الالتفافية (CNN) [19)]

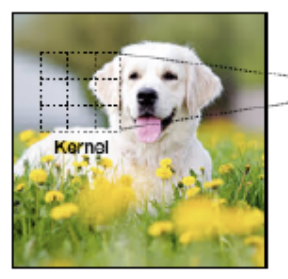

Convolution

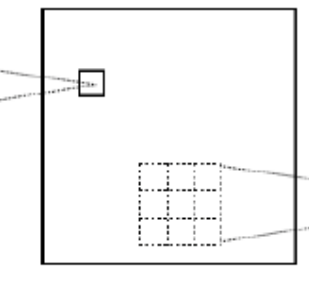

Pooling

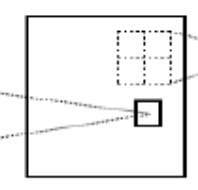

Convolution

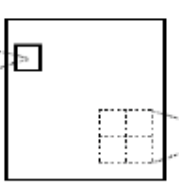

Pooling

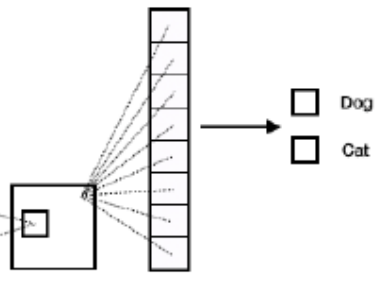

Fully-connected
9 في هذه الدراسة تم

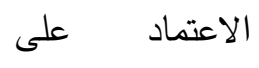
الشبكات العصبية (الشبكة العصبية (الثنة (الشبكة الالتفافية والثبكة العصبية متعددة الطبقات) لبناء نظام التعرف على المتحدث. حيث تمت بناء هيكلية الشبكات بناءً على التجربة. 1.9

تتألف هيكلية الشبكة من 4 طبقات مرتبطة ارتباطا كليا ، بالإضافة الى طبقة الادخال والتي تحتوي على 1131 خلية و3 طبقات مخفية، تحتوي الطبقة الأولى على 256 خلية اما الطبقة المخفية الثانية فتحتوي على 256 خلية بينما الطبقة المخفية الثالثة فتحتوي على 128 خلية، تم استخدام دالة التشيط relu في الطبقات المخفية لتدريب الشبكة وتحسين الاوزان، اما طبقة الإخراج فتتألف من 36 خلية وهي تمثل هذه الطبقة عدد المتحدثين، اما دالة التتشيط في طبقة الاخراج فهي Softmax. يوضح الجدول (1) معمارية الشبكة .MNN

MNN الجدول (1): معمارية الثبكة

\begin{tabular}{|c|c|c|}
\hline Layer (type) & Output Shape & Param \# \\
\hline Flatten (Flatten) & (None , 1131) & 0 \\
\hline Dense (Dense) & (None , 128) & 144896 \\
\hline Dense_1 (Dense) & (None , 128) & 16512 \\
\hline
\end{tabular}


Journal of Education and Science (ISSN 1812-125X), Vol: 30, No: 4, 2021 (141-160)

\begin{tabular}{|c|c|c|}
\hline Dense_2 (Dense) & (None , 128) & 16512 \\
\hline Dense_3 (Dense) & (None, 35) & 4515 \\
\hline
\end{tabular}

2.9

تتألف شبكة CNN من 5 طبقات ، تتكون الطبقة الأولى من طبقة التفافية ثنائية (Conv2D) ، يتم في هذه الطبقة استخدام (64) فلتر (Kernel) كل فلتر يتمثل بمصفوفة ثنائية بحجم (4*4) و دالة تتشيط نوع relu ،بالإضافة الى الطبقة الالتفافية كتم استخدام التجميع (MaxPooling2D) بحجم (3,3) والتي تعمل على تبسيط المعلومات التي تم الحصول عليها بواسطة الطبقة الالتفافية والخطوة (strides) بحجم (2,2) لتحديد موقع الفلتر في المرحلة التالية بالنسبة للمرحلة الحالية ، ونوع الحشو (padding) التي تعمل على اضافة اصفار الى البيانات المدخلة و في الاتجاهات الاربع ، تهدف هذه العملية الى جعل ابعاد الاخراج مساوية لإبعاد الادخال، ولزيادة سرعة عملية التدريب يتم استخدام دفعة التطبيع (Batch Normalization) ،اما الطبقة الثانية فهي شبيهة بالطبقة الالتفافية

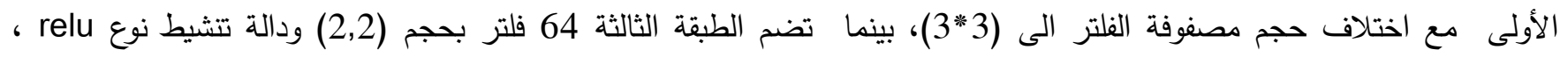
بالإضافة الى MaxPooling2D بحجم (2, 2trides بحجم (2, 2) ونوع الحشو صفري ايضا. الطبقة الرابعة هي طبقة تسطح flatten السينية softmax. الشكل يوضح الجدول (2) معمارية الشبكةCNN.كما يوضح الثكل (10)هيكلية الثبكة العصبية الالتفافية (CNN)

\section{الثكل (10): يوضح هكيلية الثبكة العصبية الالتفافية (CNN)}

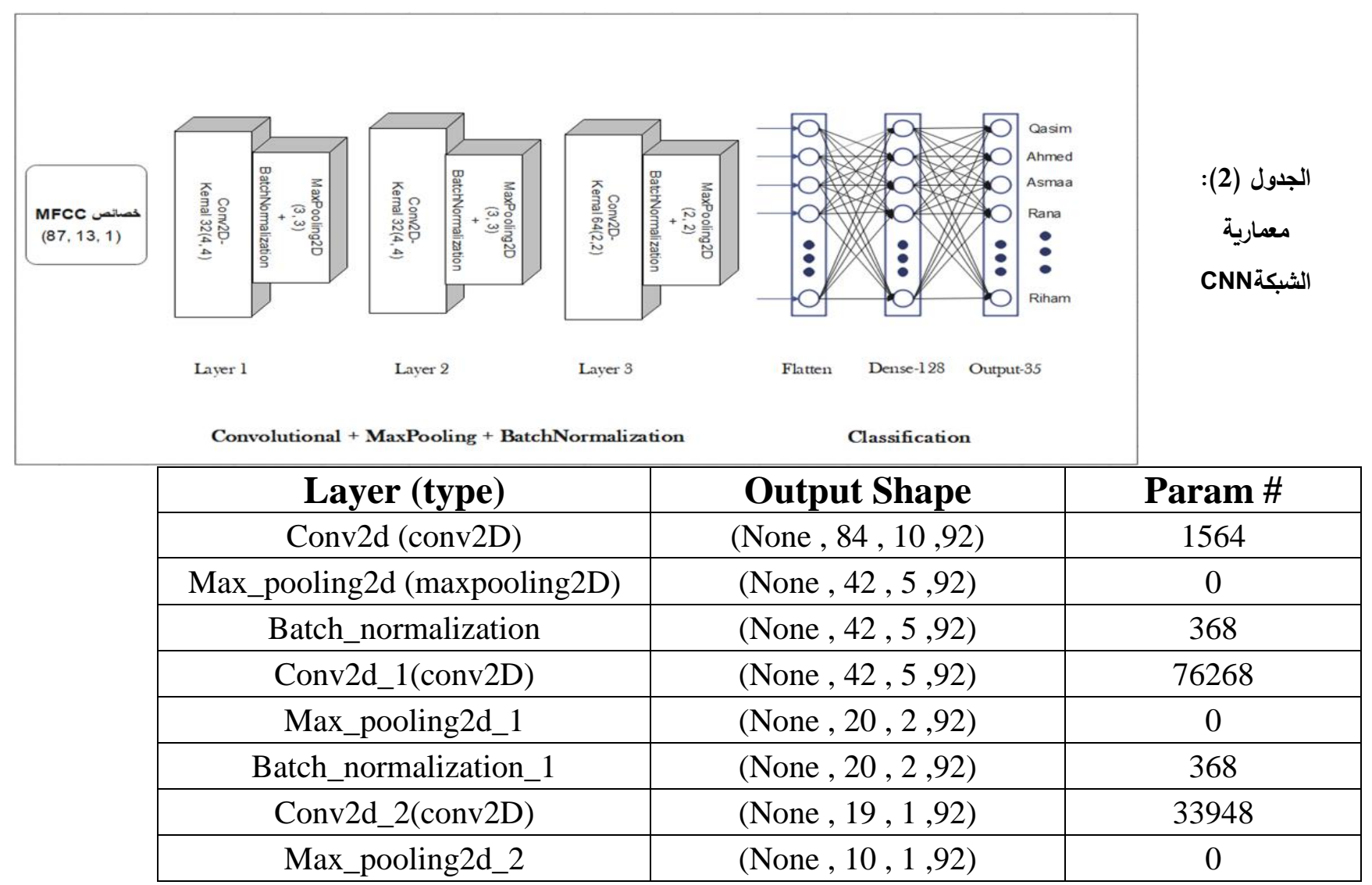




\begin{tabular}{|c|c|c|}
\hline Batch_normalization_2 & (None $, 10,1,92)$ & 368 \\
\hline Flatten_1 & (None,920) & 0 \\
\hline Dense_4 & (None,920) & 117888 \\
\hline Dense_5 & (None,920) & 4515 \\
\hline
\end{tabular}

3.9

بعد الانتهاء من اعداد هيكلية الشبكات بشكل كامل، لابد من تقسيم قاعدة البيانات الى أجزاء، قسم منها للتدريب واخر للاختبار وقسم اخر لتدقيق صحة النتائج التي تستخدم فقط مع شبكة CNN. تم تقسيم قواعد البيانات الصوتية حسب الجدول (3). الجدول (3): يوضح الية التقسم قاعدة البيانات الصوتي

\begin{tabular}{|c|c|c|c|}
\hline الشبكة & التدريب & التحقيق & الاختبار \\
\hline MNN & $\% 70$ & - & $\% 30$ \\
\hline CNN & $\% 40$ & $\% 30$ & $\% 30$ \\
\hline
\end{tabular}

4.9 التدريب تشكل مرحلة

تدريب الشبكة العصبية مرحلة أساسية وهامة تأتي بعد مرحلة اعداد هيكلية الشبكة وتقسيم البيانات، حيث يجري تدريبها على مجموعة

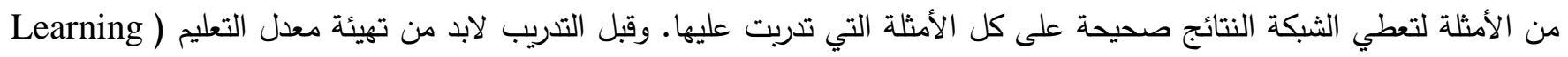

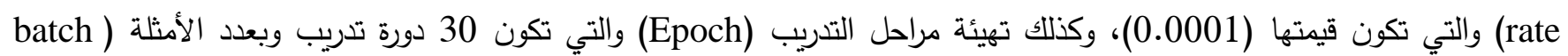
(size ) والتي تكون 10. 5.9 مرحلة الاختبار

اختبار الشبكة مشابه تماماً لعملية التدريب إلا أن الشبكة في هذه المرحلة لا تضبط أوزانها، وإنما فقط تقوم بعمليتي الجمع والتحويل ومقارنة الناتج الذي تتجه الشبكة بالناتج الهدف. حيث يتم عرض فئة اختبار على الثبكة وتحتوي هذه الفئة على مجموعة من المدخلات والمخرجات المصاحبة لكل مدخل. وبعد اكتمال مرحلة التدريب والاختبار . 9.الية عمل النظام

في البداية يتم تهيئة قاعدة البيانات ثم يتم استخلاص الخصائص وخزن النتائج في ملف القاموس Code_Dictionary، يتم اخذ

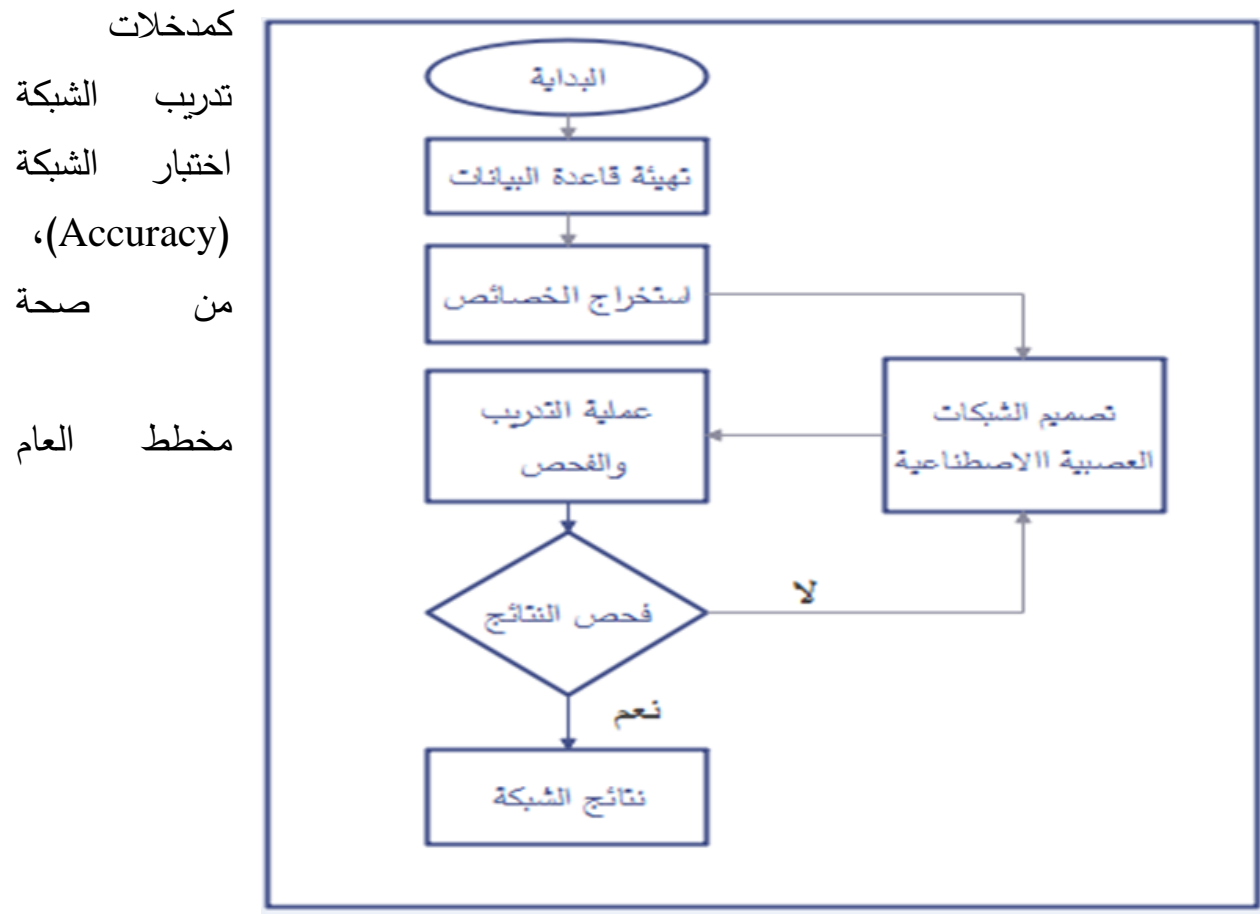
الخصائص من القاموس والتي تعتبر للشبكات العصبية, بعد ذللك يتم وضبط معاملات الثبكة واخيرا يتم والحصول على النتائج الدقة وبعد الحصول على النتائج يتم التأكد

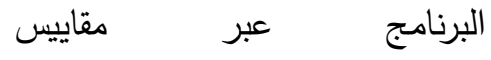
(FAR,FRR).الشكل (11) يوضح مئح

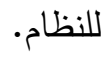


المقاييس التي نعتمد عليها في هذه الدراسة في تتبؤ الشبكة (Accuracy)، واما التحقق من صحة النظام عبر مقاييس (FARR)،

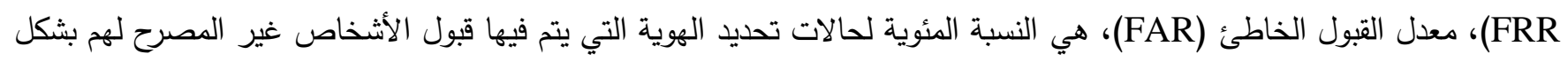

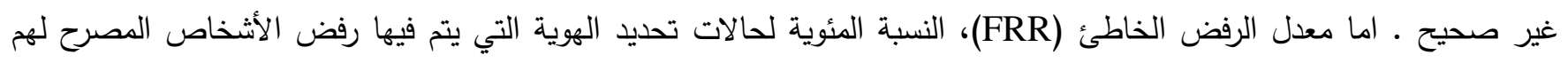
بشكل غير صحيح. توضح المعادلات التالية كلما ذكر في هذه الفقرة [17]:

Accuracy $=\frac{\text { No. of correct recognitions }}{\text { Totsl no. of trials }}$

$F R R=\frac{\text { No.of true }- \text { speakers rejected }}{\text { Total no.of true }- \text { speaker trials }}$

$$
F A R=\frac{\text { No. of impostors accepted }}{\text { Total no. of impostor attempts }}
$$

11. النتائج والمناقشة نتيجة للعملية السابقة هي تحويل البيانات الصوتية إلى العديد من ميزات الخصائص، تعتبر هذه الخصائص كمدخلات للشبكات العصبية، في الفترات التالية سيتم مناقشة النتائج النظام التي تما الحصول عليها باستخدام الثبكة العصبية الالتفافية (CNN) والشبكة الصبية متعددة الطبقات (MNN). 
تختلف النتائج لعملية التعرف على المتحدث باختلاف الشبكات العصبية وجودة قاعدة البيانات الصوتية المستخدمة لعملية التعرف على المتحدث، لذا فقد تما قياس كفاءة اداء الشبكات العصبية باستخدام مقياس الدقة (Accuracy)، وعلمية التحقق عن طريق مقاييس

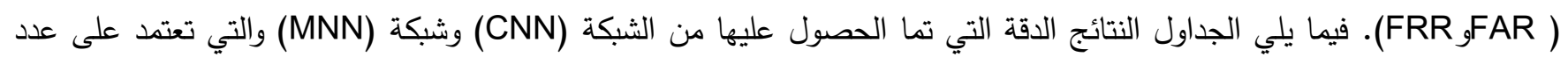

الجدول (4): النتائج الدقة للشبكات العصبية بناء على قاعدة بيانات(QS-Dataset)

\begin{tabular}{|c|c|c|}
\hline \multirow{2}{*}{ No. of Speakers } & \multicolumn{2}{|c|}{ Accuracy } \\
\cline { 2 - 3 } & MNN & CNN \\
\hline 5 & $\% 100$ & $\% 70$ \\
\hline 10 & $\% 88$ & $\% 89$ \\
\hline 15 & $\% 84$ & $\% 95$ \\
\hline 20 & $\% 85$ & $\% 96$ \\
\hline 25 & $\% 82$ & $\% 97$ \\
\hline 30 & $\% 81$ & $\% 96$ \\
\hline 35 & $\% 80$ & $\% 96$ \\
\hline
\end{tabular}

الجدول (5):النتائج الدقة للثبكات العصبية بناء على قاعدة بيانات (audioMNIST_meta)

\begin{tabular}{|c|c|c|}
\hline \multirow{2}{*}{ No. of Speakers } & \multicolumn{2}{|c|}{ Accuracy } \\
\cline { 2 - 3 } & MNN & CNN \\
\hline 5 & $\% 87$ & $\% 53$ \\
\hline 10 & $\% 76$ & $\% 79$ \\
\hline 15 & $\% 70$ & $\% 83$ \\
\hline 20 & $\% 66$ & $\% 81$ \\
\hline 25 & $\% 63$ & $\% 82$ \\
\hline 30 & $\% 56$ & $\% 83$ \\
\hline
\end{tabular}

في الجدول (4) تحتوي على نتائج الدقة (Accuracy) لعملية التعرف على هوية المتحدث باستخدام الشبكة العصبية متعددة الطبقات (MNN) والثبكة العصبية الالتفافية (CNN) بالاعتماد على قاعدة البيانات الصوتية (QS-Dataset)، نلاحظ النتائج شبكة عالية جدا بنسبة تصل الى 100\% (MNN) تستقر هذه النسبة لدرجة معينة وذلك لأنه نوعية هذه الشبكة تتعامل مع بيانات القليلة بالمقارنة مع الثبكة العصبية الالتفافية (CNN) 
تكون عكس النتائج الثبكة (MNN) حيث تكون الثبكة ذات كفاءة قليلة كلما كانت عدد البيانات قليلة بعكس ما اذا توفرت بيانات كبيرة تكون نتائج الشبكة عالية جدا ، اما النتائج الدقة في الجدول (5) تكون مختلفة عن النتائج الجدول (4) يعود ذلك الى السبب الرئيسي هو

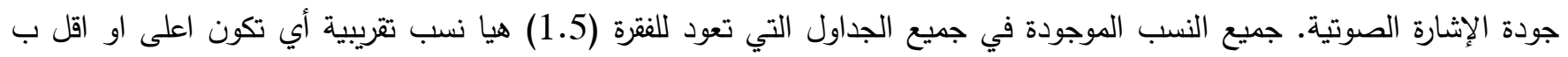
1\% الى 5\% والسبب في ذلك يعود الى القيم الأولية التي تعطى للمعاملات الثبكة بشكل تلقائي عند بداية التدريب ولكون هذه الثبكات محددة بعدد فترات تدريبية ، حيث عند كل مرحلة تدريب تعطي قيماً مختلفة للمعاملات ولضمان حصول على نسبة تقدئ تقريبية جيدة يتم تدريب الشبكات لـ 5 مرات وفي كل مرة يتم تسجيل النسبة التي تما الحصول عليها من شبكات وبعد ذلك يتم جمع جميع القيم وتقسيمها لغاليا على 5 للحصول على النسبة المتوقعة. الثكل (12) و(13) يبين النتائج الشبكات بشكل مخطط بياني .

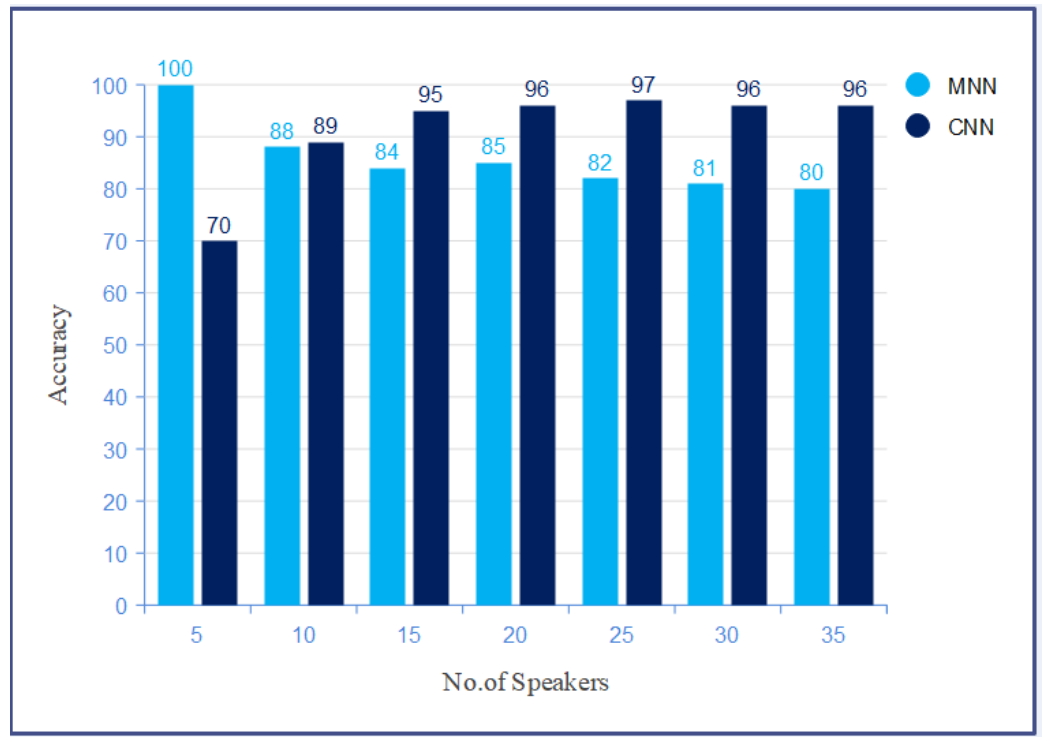

الشبكات المبنية

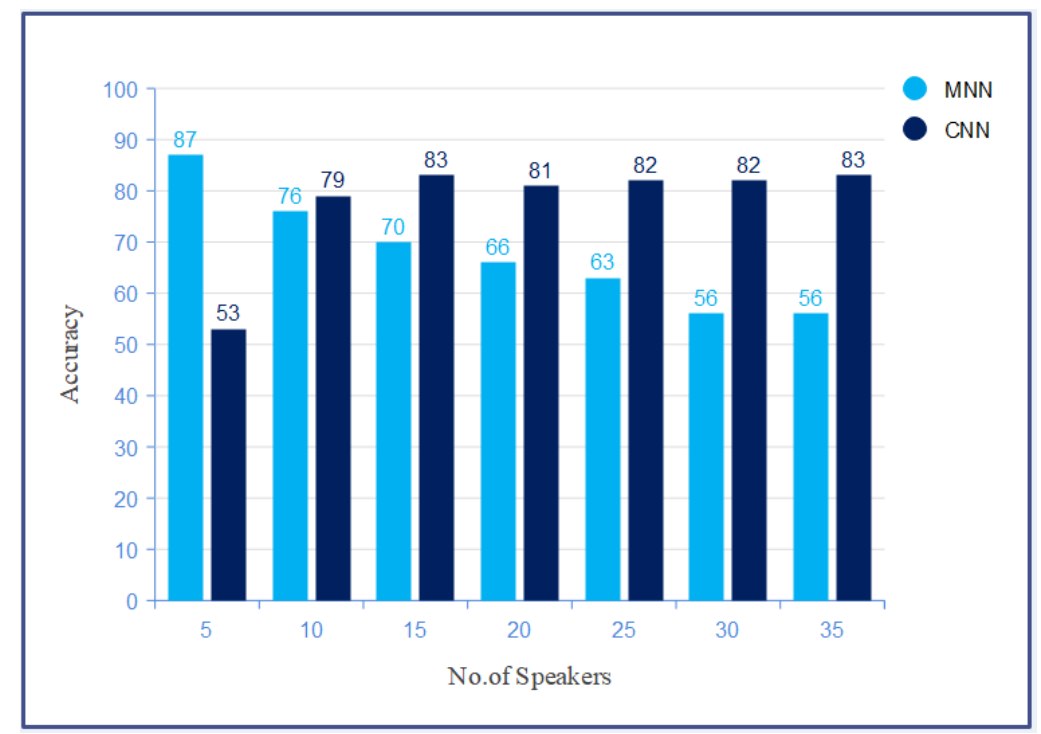

الثكل (12) مخطط بياني لنتائج

على قاعدة بيانات (QA-Dataset) 
الثكل (13) مخطط بياني لنتائج الثبكات المبنية على قاعدة بيانات (audioMNIST_meta)

2.11

بعد الحصول على النتائج الدقة (Accuracy)، لابد من التحقق من عمل النظام عن طريق مقاييس (FRR وFAR)، تم اخذ عينات صوتية من القواعد البيانات والبالغ عددها 875 مقطع صوتيهيتم اخبار النظام ان جميع هذه الأصوات تعود لمتحدث معين كيقوم النظام بالتحقق من جميع مقاطع الصوتية وبعدها تخزن الناتج ثم تكرر العملية على بقية المتحدثين والحصول على ناتج نهائي ،تسمى هذه العملية بـ معدل القبول الخاطئ (FAR).اما مقياس معدل الرفض الخاطئ (FRR)، فيؤخذ عينات صوتية تعود للمتحدث نفسه ويقوم النظام بفحص نسبة فشل النظام في التعرف على المتحدث نفسة.فيما يلي النتائج التي تم التوصل اليها :

الجدول (6): نتائج التحقق من الثبكة العصبية متعددة الطبقات (MNN)

\begin{tabular}{|c|c|c|}
\hline Dataset & FAR & FRR \\
\hline QS-Dataset & $\% 2.85$ & $\% 10.4$ \\
\hline audioMNIST_meta & $\% 2.85$ & $\% 24.34$ \\
\hline
\end{tabular}

الجدول (7): نتائج التحقق من الثبكة العصبية الالتفافية (CNN)

\begin{tabular}{|c|c|c|}
\hline Dataset & FAR & FRR \\
\hline QS-Dataset & $\% 2.85$ & $\% 3.88$ \\
\hline audioMNIST_meta & $\% 2.85$ & $\% 11.31$ \\
\hline
\end{tabular}

1.2.11 مناقشة النتائج التحقق من النظام

في الجدول (6) و (7) نلاحظ ان نسبة مقياس (FAR)هيا 2.85\% ،وسبب في ذللك عند فحص النظام وفق مقاطع الصوتية المخصصة لهذه الغرض كانت من ضمن هذه مقاطع صوتية أصوات تعود نفس الأشخاص المسجلين في النظام .اما القيم التي تعود لكقياس (FRR)،نلاحظ في الجدول (6) قيم اعلى مما هيا موجوده في الجدول (7) وسبب في ذللك يعود الى ان الأنظمة التي بنيت بالاعتماد على شبكة MNN تكون اقل دقة واقل فعالية عندما تتعامل مع البيانات الكبيرة بعكس الأنظمة التي تم بناءها بالاعتماد على شبكة CNN تكون اكثر كفاءهواكثر دقة .

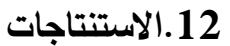

تقدم هذه الدراسة أحدث انظمة التعرف على المتحدثين والتحقق منهم، حيث اعتمد على أساليب التعلم العميق التي تتضمن الثبكات

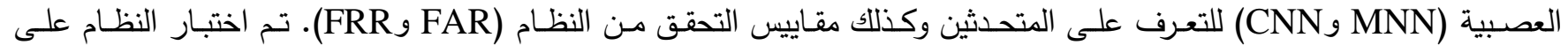
مجموعة من قواعد البيانات (audioMNIST_meta ، QS-Dataset ) ، حيث يوضح نسبة الدقة التي حصلت عليها عند استخدام قاعدة البيانات (QS-Dataset) وكذلك نسبة الدقة التي حصلت عليها عند استخدام قاعدة البيانات (audioMNIST_meta)،ويمكن 


$$
\begin{aligned}
& \text { ملاحظة عند استخدام اقل عدد ممكن من البيانات تكون شبكة MNN اكثر فعالية بينما اذا كانت كمية البيانات كبيرة تكون شبكة } \\
& \text { اكثر فعالية. يتميز الأنظمة التي تما بناءها بسرعة التدريب الشبكات وباقل وقت وبعدد قليل من عينات التدريب وكذلك ما يميز } \\
& \text { هذه الأنظمة انه لا تحتاج الى حاسبات ذات مواصفات عالية في عملية تدريب الشبكة . } \\
& \text { شكر وتقدير } \\
& \text { يتقدم الباحثون بالثكر للمراجعين والمحررين على ملاحظاتهم المفيدة. كما يتقدم الباحثون بالثكر والتقدير لقسم علوم الحاسوب، }
\end{aligned}
$$

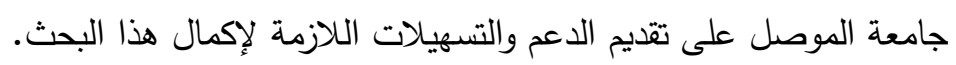

13

[1] B. Alkhatib and M. M. K. Eddin, "Voice Identification Using MFCC and Vector Quantization," Baghdad Sci. J., vol. 17, no. 3 ملحق, 2020.

[2] J. Martinez, H. Perez, E. Escamilla, and M. M. Suzuki, "Speaker recognition using Mel frequency Cepstral Coefficients (MFCC) and Vector quantization (VQ) techniques," in CONIELECOMP 2012, 22nd International Conference on Electrical Communications and Computers, 2012, pp. 248-251.

[3] S. Bunrit, T. Inkian, N. Kerdprasop, and K. Kerdprasop, “Text-Independent Speaker Identification Using Deep Learning Model of Convolution Neural Network," Int. J. Mach. Learn. Comput., vol. 9, no. 2, pp. 143-148, 2019.

[4] S. V Ault, R. J. Perez, C. A. Kimble, and J. Wang, “On speech recognition algorithms,” Int. J. Mach. Learn. Comput., vol. 8, no. 6, pp. 518-523, 2018.

[5] T. Kinnunen and H. Li, "An overview of text-independent speaker recognition: From features to supervectors," Speech Commun., vol. 52, no. 1, pp. 12-40, 2010.

[6] I. GAVAT and D. MILITARU, "New trends in machine learning for speech recognition," in Annual Symposium of the Institute of Solid Mechanics and Session of the Commission of Acoustics SISOM, At Bucharest, Romania, 2015, vol. 2015, pp. 271-276.

[7] P. Sharma and A. Garg, "Feature Extraction and Recognition of Hindi Spoken Words using Neural Networks,” Int. J. Comput. Appl., vol. 142, no. 7, pp. 12-17, 2016.

[8] A. Antony and R. Gopikakumari, "Speaker identification based on combination of MFCC and UMRT based features," Procedia Comput. Sci., vol. 143, pp. 250-257, 2018.

[9] V. Thanda Setty, "Speaker Recognition using Deep Neural Networks with reduced Complexity," 2018, M.sc. Thesis, Texas State University.

[10] S. Safavi, P. Jancovic, M. J. Russell, and M. J. Carey, "Identification of gender from children's speech by computers and humans.," in INTERSPEECH, 2013, pp. 2440-2444.

[11] R. Jagiasi, S. Ghosalkar, P. Kulal, and A. Bharambe, "CNN based speaker recognition in language and text-independent small scale system," in 2019 Third International conference on I-SMAC (IoT in Social, Mobile, Analytics and Cloud)(I-SMAC), 2019, pp. 176-179.

[12] S. Abd El-Moneim, M. A. Nassar, M. I. Dessouky, N. A. Ismail, A. S. El-Fishawy, and F. E. Abd El-Samie, "Text-independent speaker recognition using LSTM-RNN and speech enhancement," Multimed. Tools Appl., vol. 79, no. 33, pp. 24013-24028, 2020. 
[13] S. Becker, M. Ackermann, S. Lapuschkin, K.-R. Müller, and W. Samek, "Interpreting and explaining deep neural networks for classification of audio signals," arXiv Prepr. arXiv1807.03418, 2018.

[14] R. A. K. Nilu Singh, "Digital Signal Processing for Speech Signals,” Bilingual international conference of information technology, pp. 134-138.

[15] yusra faysal Ali nesaf, "Distinguishing single-pronounced Arabic numerals using a genetic algorithm," AL-Rafidain Journal of Computer Sciences and Mathematics, vol. 1(11), 2013.

[16] Kamath, Uday, John Liu, and James Whitaker. Deep learning for NLP and speech recognition. Vol. 84. Cham: Springer, 2019.

[17] Michael Negnevitsky, "Artificial Intelligence: A Guide to Intelligent Systems.," in Polyhedron, Third Edit., vol. 123, University of Tasmania: Addison Wesley, 2005, pp. 165-216.

[18] R. Yamashita, M. Nishio, R. K. G. Do, and K. Togashi, "Convolutional neural networks: an overview and application in radiology," Insights Imaging, vol. 9, no. 4, pp. 611-629, 2018.

[19] J. Van Der Donckt, "Latent representations for spoken language," 2019, M.sc. Thesis, Gent Universiteit. 